\title{
Biomass-Derived Carbon for Electrode Fabrication in Microbial Fuel Cells: A Review
}

Wei Yang ${ }^{a, *}$, and Shaowei Chen ${ }^{b, *}$

a State Key Laboratory of Hydraulics and Mountain River Engineering, College of Water Resource \& Hydropower, Sichuan University, Chengdu 610065, CHINA

E-mail: wei_yang@scu.edu.cn

${ }^{\mathrm{b}}$ Department of Chemistry and Biochemistry, University of California, 1156 High Street, Santa Cruz, California 95064, USA

E-mail: shaowei@ucsc.cn

\section{Corresponding Authors}

*E-mail: wei_yang@scu.edu.cn, Phone/Fax:+86-028-8540-5633

*E-mail: shaowei@ucsc.cn, Phone/Fax: 831-459-5841/831-459-2935

\section{Author Contributions}

The manuscript was written through contributions of all authors. All authors have given approval to the final version of the manuscript. 
Table S1 Summary of biomass-derived carbon anodes in MFCs reported in recent literature

\begin{tabular}{|c|c|c|c|c|c|c|}
\hline Anode precursor & Preparation process & $\begin{array}{l}\text { Type of } \\
\text { MFC }\end{array}$ & Inoculation & Substrate & $\begin{array}{l}\text { Maximum power } \\
\text { output }\end{array}$ & References \\
\hline Bamboo & Carbonization & $\begin{array}{l}\text { Two- } \\
\text { chamber }\end{array}$ & $\begin{array}{c}\text { Preacclimated bacteria from an } \\
\text { active MFC }\end{array}$ & Acetate & $1652 \pm 18 \mathrm{~mW} / \mathrm{m}^{2}$ & 1 \\
\hline Pinecone & Carbonization & $\begin{array}{l}\text { Two- } \\
\text { chamber }\end{array}$ & $\begin{array}{c}\text { Preacclimated bacteria from an } \\
\text { active MFC }\end{array}$ & Acetate & $10.88 \mathrm{~W} / \mathrm{m}^{3}$ & 2 \\
\hline Sewage sludge & $\begin{array}{l}\text { Methane } \\
\text { chemical vapor }\end{array}$ & $\begin{array}{l}\text { Two- } \\
\text { chamber }\end{array}$ & $\begin{array}{c}\text { Preacclimated bacteria from an } \\
\text { active MFC }\end{array}$ & Acetate & $2228 \mathrm{~mW} / \mathrm{m}^{2}$ & 3 \\
\hline Cake & $\begin{array}{c}\text { Carbonization and } \\
\text { polymerization }\end{array}$ & $\begin{array}{l}\text { Single- } \\
\text { chamber }\end{array}$ & $\begin{array}{l}\text { Preacclimated bacteria from an } \\
\text { active MFC }\end{array}$ & Acetate & $1307 \mathrm{~mW} / \mathrm{m}^{2}$ & 4 \\
\hline Bread & Carbonization & $\begin{array}{l}\text { Two- } \\
\text { chamber }\end{array}$ & $\begin{array}{l}\text { Preacclimated bacteria from activated } \\
\text { anaerobic sludge }\end{array}$ & Acetate & $134 \mathrm{~mW} / \mathrm{m}^{2}$ & 5 \\
\hline Pomelo peel & Carbonization & - & Domestic wastewater & Acetate & $4.0 \mathrm{~mA} / \mathrm{cm}^{2}$ & 6 \\
\hline Neem wood & Carbonization & $\begin{array}{l}\text { Two- } \\
\text { chamber }\end{array}$ & $\begin{array}{c}\text { Preacclimated bacteria from an } \\
\text { active MFC }\end{array}$ & Glucose & $256 \pm 25 \mathrm{~mW} / \mathrm{m}^{2}$ & 7 \\
\hline Cocklebur fruit & Carbonization & $\begin{array}{l}\text { Single- } \\
\text { chamber }\end{array}$ & $\begin{array}{c}\text { Preacclimated bacteria from an } \\
\text { active MFC }\end{array}$ & Acetate & $572.57 \pm 24.90 \mu \mathrm{W}$ & 8 \\
\hline Silk cocoon & Carbonization & $\begin{array}{l}\text { Single- } \\
\text { chamber }\end{array}$ & $\begin{array}{l}\text { Preacclimated bacteria from activated } \\
\text { anaerobic sludge }\end{array}$ & Acetate & $\sim 5.0 \mathrm{~mW} / \mathrm{g}$ & 9 \\
\hline Loofah Sponge & $\begin{array}{l}\text { Polymerization and } \\
\text { carbonization }\end{array}$ & $\begin{array}{l}\text { Single- } \\
\text { chamber }\end{array}$ & $\begin{array}{l}\text { Preacclimated bacteria from an } \\
\text { active MFC }\end{array}$ & Acetate & $1090 \pm 72 \mathrm{~mW} / \mathrm{m}^{2}$ & 10 \\
\hline Municipal sludge & Carbonization and & Two- & Shewanella oneidensis MR-1 & Lactate & $568.5 \mathrm{~mW} / \mathrm{m}^{2}$ & 11 \\
\hline
\end{tabular}




\begin{tabular}{|c|c|c|c|c|c|c|}
\hline & polymerization & chamber & & & & \\
\hline Cotton textile & $\begin{array}{l}\text { Carbonization and } \\
\text { polymerization }\end{array}$ & $\begin{array}{l}\text { Single- } \\
\text { chamber }\end{array}$ & $\begin{array}{c}\text { Preacclimated bacteria from an } \\
\text { active MFC }\end{array}$ & Acetate & $931 \pm 61 \mathrm{~mW} / \mathrm{m}^{2}$ & 12 \\
\hline kapok fibers & Carbonization & $\begin{array}{l}\text { Single- } \\
\text { chamber }\end{array}$ & $\begin{array}{c}\text { Preacclimated bacteria from anaerobic } \\
\text { sludge }\end{array}$ & Acetate & $1738.1 \mathrm{~mW} / \mathrm{m}^{2}$ & 13 \\
\hline King mushroom & Carbonization & - & $\begin{array}{l}\text { Anaerobic digester of a } \\
\text { sewage treatment plant }\end{array}$ & Acetate & $2.09 \mathrm{~mA} / \mathrm{cm}^{2}$ & 14 \\
\hline Wild mushroom & Carbonization & $\longrightarrow$ & $\begin{array}{l}\text { Anaerobic digester of a } \\
\text { sewage treatment plant }\end{array}$ & Acetate & $3.02 \mathrm{~mA} / \mathrm{cm}^{2}$ & 14 \\
\hline Corn stem & Carbonization & - & $\begin{array}{l}\text { Anaerobic digester of a } \\
\text { sewage treatment plant }\end{array}$ & Acetate & $3.12 \mathrm{~mA} / \mathrm{cm}^{2}$ & 14 \\
\hline Sewage sludge & Carbonization & $\begin{array}{l}\text { Two- } \\
\text { chamber }\end{array}$ & $\longrightarrow$ & Acetate & $3.2 \mathrm{~W} / \mathrm{m}^{2}$ & 15 \\
\hline Sugarcane & Carbonization & $\begin{array}{l}\text { Two- } \\
\text { chamber }\end{array}$ & $\begin{array}{c}\text { Preacclimated bacteria from an } \\
\text { active MFC }\end{array}$ & Acetate & $59.94 \pm 2.81 \mathrm{~W} / \mathrm{m}^{3}$ & 16 \\
\hline $\begin{array}{c}\text { Sewage sludge/coconut } \\
\text { shell }\end{array}$ & Carbonization & $\begin{array}{l}\text { Single- } \\
\text { chamber }\end{array}$ & $\begin{array}{c}\text { Preacclimated bacteria from an } \\
\text { active MFC }\end{array}$ & Acetate & $1069 \pm 15 \mathrm{~mW} / \mathrm{m}^{2}$ & 17 \\
\hline Loofah sponges & Chinese ink-coating & $\begin{array}{l}\text { Single- } \\
\text { chamber }\end{array}$ & $\begin{array}{c}\text { Preacclimated bacteria from an } \\
\text { active MFC }\end{array}$ & Acetate & $0.82 \mathrm{~mW} / \mathrm{cm}^{3}$ & 18 \\
\hline Loofah sponge & Carbon black-coating & $\begin{array}{l}\text { Two- } \\
\text { chamber }\end{array}$ & $\begin{array}{c}\text { Preacclimated bacteria from an } \\
\text { active MFC }\end{array}$ & Acetate & $61.7 \pm 0.6 \mathrm{~W} / \mathrm{m}^{3}$ & 19 \\
\hline Chestnut shell & $\begin{array}{l}\text { Carbonization and } \\
\text { activation }\end{array}$ & $\begin{array}{l}\text { Single- } \\
\text { chamber }\end{array}$ & Municipal wastewater & Acetate & $23.6 \mathrm{~W} / \mathrm{m}^{3}$ & 20 \\
\hline kenaf & Carbonization & - & Primary domestic wastewater & Acetate & $32.5 \mathrm{~A} / \mathrm{m}^{2}$ & 21 \\
\hline Chestnut shell & $\begin{array}{l}\text { Carbonization and } \\
\text { activation }\end{array}$ & $\begin{array}{l}\text { Single- } \\
\text { chamber }\end{array}$ & $\begin{array}{c}\text { Preacclimated bacteria from an } \\
\text { active MFC }\end{array}$ & Acetate & $759 \pm 38 \mathrm{~mW} / \mathrm{m}^{2}$ & 22 \\
\hline
\end{tabular}


Table S2 Summary of biomass-derived ORR catalysts in MFCs in recent literature

\begin{tabular}{|c|c|c|c|c|c|c|}
\hline Cathode precursor & Preparation procedure & Type of MFC & $\begin{array}{l}\text { Support } \\
\text { substrate }\end{array}$ & $\begin{array}{l}\text { Catalyst } \\
\text { loading }\end{array}$ & $\begin{array}{c}\text { Maximum power } \\
\text { output }\end{array}$ & References \\
\hline Cornstalk & Hydrothermal and carbonization & $\begin{array}{l}\text { Single- } \\
\text { chamber }\end{array}$ & SSM & - & $1122 \pm 32 \mathrm{~mW} / \mathrm{m}^{2}$ & 23 \\
\hline Corncob & Carbonization & $\begin{array}{l}\text { Single- } \\
\text { chamber }\end{array}$ & Carbon cloth & $0.5 \mathrm{mg} \mathrm{cm}^{-3}$ & $458.85 \mathrm{~mW} / \mathrm{m}^{2}$ & 24 \\
\hline Sugarcane refuse & Activation and carbonization & Two-chamber & SSM & - & $120 \pm 15 \mathrm{~mW} / \mathrm{m}^{2}$ & 25 \\
\hline Green foxtail & Carbonization & $\begin{array}{l}\text { Single- } \\
\text { chamber }\end{array}$ & SSM & $10 \mathrm{mg} \mathrm{cm}^{-2}$ & $665 \mathrm{~mW} / \mathrm{m}^{2}$ & 26 \\
\hline Cornstalk & Carbonization & $\begin{array}{l}\text { Single- } \\
\text { chamber }\end{array}$ & SSM & $\longrightarrow$ & $1003 \mathrm{~mW} / \mathrm{m}^{2}$ & 27 \\
\hline Pectin & Carbonization and template method & $\begin{array}{l}\text { Single- } \\
\text { chamber }\end{array}$ & SSM & - & $1161.13 \mathrm{~mW} / \mathrm{m}^{2}$ & 28 \\
\hline Dandelion seed & Activation and carbonization & $\begin{array}{l}\text { Single- } \\
\text { chamber }\end{array}$ & Carbon cloth & $2 \mathrm{mg} \mathrm{cm}^{-2}$ & $975 \pm 46 \mathrm{~mW} / \mathrm{m}^{2}$ & 29 \\
\hline Bamboo & Carbonization & $\begin{array}{l}\text { Single- } \\
\text { chamber }\end{array}$ & SSM & $50 \mathrm{mg} \mathrm{cm}^{-2}$ & $\begin{array}{c}1056 \\
\pm 38 \mathrm{~mW} / \mathrm{m}^{2}(1719 \\
\left. \pm 82 \mathrm{~mW} / \mathrm{m}^{2}\right)\end{array}$ & 30 \\
\hline Egg & Carbonization & $\begin{array}{l}\text { Single- } \\
\text { chamber }\end{array}$ & Carbon cloth & $0.5 \mathrm{mg} \mathrm{cm}^{-3}$ & $737.1 \mathrm{~mW} / \mathrm{m}^{2}$ & 31 \\
\hline Alfalfa Leaf & Carbonization and activation & $\begin{array}{l}\text { Single- } \\
\text { chamber }\end{array}$ & Carbon cloth & - & $1328.9 \mathrm{~mW} / \mathrm{m}^{2}$ & 32 \\
\hline Chlorella pyrenoidosa & Carbonization & Single- & SSM & $10 \mathrm{mg} \mathrm{cm}^{-2}$ & $2068 \pm 30 \mathrm{~mW} / \mathrm{m}^{2}$ & 33 \\
\hline
\end{tabular}




\begin{tabular}{|c|c|c|c|c|c|c|}
\hline & & chamber & & & & \\
\hline Kraft paper & Carbonization & $\begin{array}{l}\text { Single- } \\
\text { chamber }\end{array}$ & - & $1 \mathrm{mg} \mathrm{cm}^{-2}$ & $830 \pm 15 \mathrm{~mW} / \mathrm{m}^{2}$ & 34 \\
\hline Bamboo tube & Carbonization & $\begin{array}{l}\text { Single- } \\
\text { chamber }\end{array}$ & 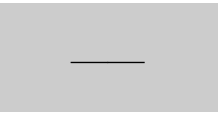 & $80 \mathrm{mg} \mathrm{cm}^{-2}$ & $40.4 \pm 1.5 \mathrm{~W} / \mathrm{m}^{3}$ & 35 \\
\hline Cocoon silk & Carbonization and activation & $\begin{array}{l}\text { Single- } \\
\text { chamber }\end{array}$ & Carbon cloth & $2 \mathrm{mg} \mathrm{cm}^{-2}$ & $800 \mathrm{~mW} / \mathrm{m}^{2}$ & 36 \\
\hline Spider silk & Carbonization and activation & $\begin{array}{l}\text { Single- } \\
\text { chamber }\end{array}$ & - & $2 \mathrm{mg} \mathrm{cm}^{-2}$ & $1800 \pm 82 \mathrm{~mW} / \mathrm{m}^{2}$ & 37 \\
\hline $\begin{array}{l}\text { Livestock sewage } \\
\text { sludge }\end{array}$ & Carbonization and activation & $\begin{array}{l}\text { Single- } \\
\text { chamber }\end{array}$ & Carbon cloth & - & $1273 \pm 3 \mathrm{~mW} / \mathrm{m}^{2}$ & 38 \\
\hline Plant moss & Hydrothermal and carbonization & $\begin{array}{l}\text { Single- } \\
\text { chamber }\end{array}$ & $\longrightarrow$ & $2 \mathrm{mg} \mathrm{cm}^{-2}$ & $703 \pm 16 \mathrm{~mW} / \mathrm{m}^{2}$ & 39 \\
\hline Bananas & Hydrothermal and carbonization & $\begin{array}{l}\text { Single- } \\
\text { chamber }\end{array}$ & Carbon cloth & - & $528.2 \mathrm{~mW} / \mathrm{m}^{2}$ & 40 \\
\hline chitin & $\begin{array}{l}\text { Hydrothermal, carbonization and } \\
\text { activation }\end{array}$ & $\begin{array}{l}\text { Single- } \\
\text { chamber }\end{array}$ & Carbon cloth & $\sim 7 \mathrm{mg} \mathrm{cm}^{-2}$ & $705 \pm 5 \mathrm{~mW} / \mathrm{m}^{2}$ & 41 \\
\hline Watermelon rind & Carbonization & $\begin{array}{l}\text { Single- } \\
\text { chamber }\end{array}$ & Carbon cloth & $0.5 \mathrm{mg} \mathrm{cm}^{-2}$ & $0.262 \mathrm{~W} / \mathrm{m}^{3}$ & 42 \\
\hline Onion & Carbonization and activation & $\begin{array}{l}\text { Single- } \\
\text { chamber }\end{array}$ & Carbon cloth & $\sim 7 \mathrm{mg} \mathrm{cm}^{-2}$ & $742 \pm 17 \mathrm{~mW} / \mathrm{m}^{2}$ & 43 \\
\hline Glucose & Hydrothermal and carbonization & $\begin{array}{l}\text { Single- } \\
\text { chamber }\end{array}$ & SSM & $20 \mathrm{mg} \mathrm{cm}^{-2}$ & $967 \pm 34 \mathrm{~mW} / \mathrm{m}^{2}$ & 44 \\
\hline Lotus leaves & Hydrothermal and carbonization & $\begin{array}{l}\text { Single- } \\
\text { chamber }\end{array}$ & - & - & $511.5 \pm 25.6 \mathrm{~mW} / \mathrm{m}^{2}$ & 45 \\
\hline Sewage sludge & Carbonization & Single- & Carbon cloth & $5 \mathrm{mg} \mathrm{cm}^{-2}$ & $500 \pm 17 \mathrm{~mW} / \mathrm{m}^{2}$ & 46 \\
\hline
\end{tabular}




\section{REFERENCES}

1. Zhang, J.; Li, J.; Ye, D.; Zhu, X.; Liao, Q.; Zhang, B., Tubular bamboo charcoal for anode in microbial fuel cells. J. Power Sources $2014,272,277-282$.

2. Wang, R. W.; Liu, D.; Yan, M.; Zhang, L.; Chang, W.; Sun, Z. Y.; Liu, S. Q.; Guo, C. S., Three-dimensional high performance free-standing anode by one-step carbonization of pinecone in microbial fuel cells. Bioresour. Technol. 2019, 292, 121956.

3. Feng, H. J.; Jia, Y. F.; Shen, D. S.; Zhou, Y. Y.; Chen, T.; Chen, W.; Ge, Z. P.; Zheng, S. T.; Wang, M. Z., The effect of chemical vapor deposition temperature on the performance of binder-free sewage sludge-derived anodes in microbial fuel cells. Sci. Total Environ. 2018, 635, 45-52.

4. Yuan, H. R.; Dong, G.; Li, D. N. A.; Deng, L. F.; Cheng, P.; Chen, Y., Steamed cake-derived 3D carbon foam with surface anchored carbon nanoparticles as freestanding anodes for high-performance microbial fuel cells. Sci. Total Environ. 2018, 636, 1081-1088.

5. Zhang, L. J.; He, W. H.; Yang, J. C.; Sun, J. Q.; Li, H. D.; Han, B.; Zhao, S. L.; Shi, Y. A.; Feng, Y. J.; Tang, Z. Y.; Liu, S. Q., Bread-derived 3D macroporous carbon foams as high performance free-standing anode in microbial fuel cells. Biosens Bioelectron 2018, 122, $217-223$.

6. Chen, S.; Liu, Q.; He, G.; Zhou, Y.; Hanif, M.; Peng, X.; Wang, S.; Hou, H., Reticulated carbon foam derived from a sponge-like natural product as a highperformance anode in microbial fuel cells. J. Mater. Chem. 2012, 22, (35), 18609-18613.

7. Senthilkumar, N.; Pannipara, M.; Al-Sehemi, A. G.; Kumar, G. G., PEDOT/NiFe2O4 nanocomposites on biochar as a free-standing anode for high-performance and durable microbial fuel cells. New J. Chem. 2019, 43, (20), 7743-7750.

8. Yai, C. C.; Chen, M. J.; Qian, Y. J.; Zhang, L.; Lu, M.; Xie, X. J.; Huang, L.; Huang, W., Packed anode derived from cocklebur fruit for improving long-term performance of microbial fuel cells. Science China-Materials 2019, 62, (5), 645-652.

9. Lu, M.; Qian, Y.; Yang, C.; Huang, X.; Li, H.; Xie, X.; Huang, L.; Huang, W., Nitrogen-enriched pseudographitic anode derived from silk cocoon with tunable flexibility for microbial fuel cells. Nano Energy 2016, 32, 382-388.

10. Yuan, Y.; Zhou, S.; Liu, Y.; Tang, J., Nanostructured macroporous bioanode based on polyaniline-modified natural loofah sponge for high-performance microbial fuel cells. Environ Sci Techno/ 2013, 47, (24), 14525-14532.

11. Ma, X. X.; Feng, C. H.; Zhou, W. J.; Yu, H., Municipal sludge-derived carbon anode with nitrogen- and oxygen-containing functional groups for high-performance microbial fuel cells. J. Power Sources 2016, 307, 105-111. 
12. Zeng, L. Z.; Zhao, S. F.; He, M., Macroscale porous carbonized polydopamine-modified cotton textile for application as electrode in microbial fuel cells. J. Power Sources 2018, 376, 33-40.

13. Zhu, H. L.; Wang, H. M.; Li, Y. Y.; Bao, W. Z.; Fang, Z. Q.; Preston, C.; Vaaland, O.; Ren, Z. Y.; Hu, L. B., Lightweight, conductive hollow fibers from nature as sustainable electrode materials for microbial energy harvesting. Nano Energy 2014, 10, 268-276.

14. Karthikeyan, R.; Wang, B.; Xuan, J.; Wong, J. W.; Lee, P. K.; Leung, M. K., Interfacial electron transfer and bioelectrocatalysis of carbonized plant material as effective anode of microbial fuel cell. Electrochim. Acta 2015, 157, 314-323.

15. Jia, Y. F.; Feng, H. J.; Shen, D. S.; Zhou, Y. Y.; Chen, T.; Wang, M. Z.; Chen, W.; Ge, Z. P.; Huang, L. J.; Zheng, S. T., High-performance microbial fuel cell anodes obtained from sewage sludge mixed with fly ash. J. Hazard. Mater. 2018, 354, 27-32.

16. Zhou, Y. H.; Zhou, G. W.; Yin, L.; Guo, J. Y.; Wan, X. K.; Shi, H. X., High-Performance Carbon Anode Derived from Sugarcane for Packed Microbial Fuel Cells. Chemelectrochem 2017, 4, (1), 168-174.

17. Yuan, Y.; Liu, T.; Fu, P.; Tang, J.; Zhou, S., Conversion of sewage sludge into high-performance bifunctional electrode materials for microbial energy harvesting. J Mater Chem A 2015, 3, (16), 8475-8482.

18. Zhou, L. H.; Sun, L. H.; Fu, P.; Yang, C. L.; Yuan, Y., Carbon nanoparticles of Chinese ink-wrapped natural loofah sponge: a low-cost three-dimensional electrode for high-performance microbial energy harvesting. J Mater Chem A 2017, 5, (28), 14741-14747.

19. Zheng, J. L.; Cheng, C. X.; Zhang, J.; Wu, X. H., Appropriate mechanical strength of carbon black-decorated loofah sponge as anode material in microbial fuel cells. Int. J. Hydrogen Energy 2016, 41, (48), 23156-23163.

20. Chen, Q.; Pu, W. H.; Hou, H. J.; Hu, J. P.; Liu, B. C.; Li, J. F.; Cheng, K.; Huang, L.; Yuan, X. Q.; Yang, C. Z.; Yang, J. K., Activated microporous-mesoporous carbon derived from chestnut shell as a sustainable anode material for high performance microbial fuel cells. Bioresour. Technol. 2018, 249, 567-573.

21. Chen, S.; He, G.; Hu, X.; Xie, M.; Wang, S.; Zeng, D.; Hou, H.; Schröder, U., A three-dimensionally ordered macroporous carbon derived from a natural resource as anode for microbial bioelectrochemical systems. ChemSusChem 2012, 5, (6), 1059-1063.

22. Chen, S. S.; Tang, J. H.; Jing, X. Y.; Liu, Y.; Yuan, Y.; Zhou, S. G., A hierarchically structured urchin-like anode derived from chestnut shells for microbial energy harvesting. Electrochim. Acta 2016, 212, 883-889.

23. Sun, Y.; Duan, Y. Q.; Hao, L.; Xing, Z. P.; Dai, Y.; Li, R.; Zou, J. L., Cornstalk-Derived Nitrogen-Doped Partly Graphitized Carbon as Efficient Metal-Free Catalyst for Oxygen Reduction Reaction in Microbial Fuel Cells. ACS App/ Mater Interfaces 2016, 8, (39), 25923-25932.

24. Li, M.; Zhang, H. G.; Xiao, T. F.; Wang, S. D.; Zhang, B. P.; Chen, D. Y.; Su, M. H.; Tang, J. F., Low-cost biochar derived from corncob as oxygen reduction catalyst in air cathode microbial fuel cells. Electrochim. Acta 2018, 283, 780-788. 
25. Bose, D.; Sridharan, S.; Dhawan, H.; Vijay, P.; Gopinath, M., Biomass derived activated carbon cathode performance for sustainable power generation from Microbial Fuel Cells. Fue/ 2019, 236, 325-337.

26. Wang, X. H.; Gong, X. B.; Peng, L.; Yang, Z.; Liu, Y., Tubular nitrogen-doped carbon materials derived from green foxtail as a metal-free electrocatalyst in microbial fuel cells for efficient electron generation. Bioelectrochemistry 2019, 127, 104-112.

27. Jing, B. J.; You, S. J.; Ma, Y. Y.; Xing, Z. P.; Chen, H.; Dai, Y.; Zhang, C. Y.; Ren, N. Q.; Zou, J. L., Fe3Se4/FeSe heterojunctions in cornstalk-derived N-doped carbon framework enhance charge transfer and cathodic oxygen reduction reaction to boost bio-electricity generation. App/ Catal B-environ $2019,244,465-474$.

28. Ma, Y. Y.; You, S. J.; Ling, B. J.; Xing, Z. P.; Chen, H.; Dai, Y.; Zhang, C. Y.; Ren, N. Q.; Zou, J. L., Biomass pectin-derived N, S-enriched carbon with hierarchical porous structure as a metal-free catalyst for enhancing bio-electricity generation. Int. J. Hydrogen Energy 2019, 44, (31), 16624-16638.

29. Tang, J. H.; Wang, Y. J.; Zhao, W. Q.; Zeng, R. J. X.; Liu, T.; Zhou, S. G., Biomass-derived hierarchical honeycomb-like porous carbon tube catalyst for the metalfree oxygen reduction reaction. J. Electroanal. Chem. 2019, 847, 113230.

30. Yang, W.; Li, J.; Ye, D.; Zhu, X.; Liao, Q., Bamboo charcoal as a cost-effective catalyst for an air-cathode of microbial fuel cells. Electrochim. Acta 2017, 224, 585592.

31. Lu, Y.; Zhu, N. W.; Yin, F. H.; Yang, T. T.; Wu, P. X.; Dang, Z.; Liu, M. L.; Wei, X. R., Biomass-derived heteroatoms-doped mesoporous carbon for efficient oxygen reduction in microbial fuel cells. Biosens Bioelectron 2017, 98, 350-356.

32. Deng, L.; Yuan, Y.; Zhang, Y.; Wang, Y.; Chen, Y.; Yuan, H.; Chen, Y., Alfalfa leaf-derived porous heteroatom-doped carbon materials as efficient cathodic catalysts in microbial fuel cells. Acs Sustain Chem Eng 2017, 5, (11), 9766-9773.

33. Fan, Z.; Li, J.; Zhou, Y.; Fu, Q.; Yang, W.; Zhu, X.; Liao, Q., A green, cheap, high-performance carbonaceous catalyst derived from Chlorella pyrenoidosa for oxygen reduction reaction in microbial fuel cells. Int. J. Hydrogen Energy 2017, 42, (45), 27657-27665.

34. Yang, W.; Li, J.; Fu, Q.; Zhang, L.; Zhu, X.; Liao, Q., A simple method for preparing a binder-free paper-based air cathode for microbial fuel cells. Bioresour. Technol. 2017, 241, 325-331.

35. Yang, W.; Li, J.; Zhang, L.; Zhu, X.; Liao, Q., A monolithic air cathode derived from bamboo for microbial fuel cells. RSC Adv 2017, 7, (45), 28469-28475.

36. Fu, P.; Zhou, L. H.; Sun, L. H.; Huang, B. H.; Yuan, Y., Nitrogen-doped porous activated carbon derived from cocoon silk as a highly efficient metal-free electrocatalyst for the oxygen reduction reaction. RSC Adv 2017, 7, (22), 13383-13389.

37. Zhou, L.; Fu, P.; Cai, X.; Zhou, S.; Yuan, Y., Naturally derived carbon nanofibers as sustainable electrocatalysts for microbial energy harvesting: A new application of spider silk. Appl. Catal B-Environ. 2016, 188, 31-38.

38. Deng, L.; Yuan, H.; Cai, X.; Ruan, Y.; Zhou, S.; Chen, Y.; Yuan, Y., Honeycomb-like hierarchical carbon derived from livestock sewage sludge as oxygen reduction 
reaction catalysts in microbial fuel cells. Int. J. Hydrogen Energy 2016, 41, (47), 22328-22336.

39. Zhou, L.; Fu, P.; Wen, D.; Yuan, Y.; Zhou, S., Self-constructed carbon nanoparticles-coated porous biocarbon from plant moss as advanced oxygen reduction catalysts. App/ Catal B-environ 2016, 181, 635-643.

40. Yuan, H. R.; Deng, L. F.; Qi, Y. J.; Kobayashi, N.; Tang, J. H., Nonactivated and Activated Biochar Derived from Bananas as Alternative Cathode Catalyst in Microbial Fuel Cells. Scientific World Journa/ 2014, 2014, 1-8.

41. Yuan, H. R.; Deng, L. F.; Cai, X. X.; Zhou, S. G.; Chen, Y.; Yuan, Y., Nitrogen-doped carbon sheets derived from chitin as non-metal bifunctional electrocatalysts for oxygen reduction and evolution. RSC Adv 2015, 5, (69), 56121-56129.

42. Zhong, K. Q.; Li, M.; Yang, Y.; Zhang, H. G.; Zhang, B. P.; Tang, J. F.; Yan, J.; Su, M. H.; Yang, Z. Q., Nitrogen-doped biochar derived from watermelon rind as oxygen reduction catalyst in air cathode microbial fuel cells. App/ Energ 2019, 242, 516-525.

43. Li, D.; Deng, L.; Yuan, H.; Dong, G.; Chen, J.; Zhang, X.; Chen, Y.; Yuan, Y., N, P-doped mesoporous carbon from onion as trifunctional metal-free electrode modifier for enhanced power performance and capacitive manner of microbial fuel cells. Electrochim. Acta 2017, 262, $297-305$.

44. Tian, X. Y.; Zhou, M. H.; Tan, C. L.; Li, M.; Liang, L.; Li, K. R.; Su, P., KOH activated N-doped novel carbon aerogel as efficient metal-free oxygen reduction catalyst for microbial fuel cells. Chem. Eng. J. 2018, 348, 775-785.

45. Ye, W. Y.; Tang, J. H.; Wang, Y. J.; Cai, X. X.; Liu, H. W.; Lin, J. Y.; Van der Bruggen, B.; Zhou, S. G., Hierarchically structured carbon materials derived from lotus leaves as efficient electrocatalyst for microbial energy harvesting. Sci. Total Environ. 2019, 666, 865-874.

46. Yuan, Y.; Yuan, T.; Wang, D.; Tang, J.; Zhou, S., Sewage sludge biochar as an efficient catalyst for oxygen reduction reaction in an microbial fuel cell. Bioresour. Technol. 2013, 144, 115-120. 